\title{
Neumología AC/DC (antes y después del COVID-19)
}

\section{Pulmonology AC/DC (before and after COVID-19)}

\author{
José Luis Sandoval Gutiérrez*
}

*Instituto Nacional de Enfermedades Respiratorias Ismael Cosío Villegas, Ciudad de México.

La Neumología como especialidad tiene siglos de haber nacido, ${ }^{1}$ a principios del siglo XX se enfocó en combatir la epidemia de tuberculosis pulmonar (TBP), lo cual llevó a la construcción de hospitales dedicados a este fin, donde la Tisiología llegó a ser confundida como el tronco de esta especialidad; para llevar un mejor control de la TBP se decidió eliminar los centros médicos formados para este fin y se convirtieron en hospitales generales, lo cual hizo que la comunidad definiera a la Neumología como «la especialidad que va a desaparecer» siendo el único que sobrevivió a su origen en nuestro país el actual Instituto Nacional de Enfermedades Respiratorias Ismael Cosío Villegas, antes Sanatorio para Tuberculosos de Huipulco. ${ }^{2}$

Personalmente, cuando decidí estudiar como tercera especialidad esta área del saber, me enfrenté a la opinión de colegas que trataban de desmotivar mi decisión por el poco futuro que albergaban a la misma hace dos décadas $y$, con el riesgo de que iba a desaparecer, yo contrargumentaba que una especialidad sería vigente mientras que los pacientes de la misma persistieran y yo contestaba «cada vez hay más neumópatas».

En la actualidad, la TBP no representa más del 5\% de los ingresos del Instituto, enfermedades como cáncer de pulmón, enfermedad pulmonar obstructiva crónica (EPOC), trastornos del dormir, asma, neumonías, influenza, enfermedades pleurales, neumopatías del paciente con $\mathrm{VIH}$, afecciones de pared torácica, cirugía de tórax, afección del

Correspondencia:

Dr. José Luis Sandoval Gutiérrez

Instituto Nacional de Enfermedades Respiratorias

Ismael Cosío Villegas, Ciudad de México.

Correo electrónico: sandovalgutierrez@gmail.com

Trabajo recibido: 29-VI-2020; aceptado: 03-VII-2020.

Citar como: Sandoval GJL. Neumología AC/DC (antes y después del COVID-19). Neumol Cir Torax. 2020; 79 (4): 287-288. https://dx.doi. org/10.35366/97972 aparato de vía área superior (otorrinolaringología), enfermedades alérgicas e inmunidad y padecimientos pediátricos (neumopediatría), han extendido el catálogo de atención a los pacientes.

Todo esto ha llevado a que la Neumología sea una especialidad de mayor relevancia en la atención de enfermos agudos y crónicos.

Las enfermedades del aparato pulmonar son la primera causa de muerte en el mundo, ${ }^{3}$ aunque esto no se haya puntualizado en muchos líderes sanitarios.

La aparición del COVID-194 ocasiona que nuestra especialidad se haya tornado atractiva, buscada, reconocida y admirada, siendo esto un acto de justicia y de necesidad colectiva ante esta nueva pandemia.

De la mano con médicos de diferentes especialidades (Medicina Interna, Infectología, Urgenciólogos e Intensivistas), se ha podido, junto con el personal de salud, afrontar esta emergencia respiratoria.

La Neumología tuvo un resurgimiento en este siglo por la otra pandemia de influenza A H1N1 en el 2009, pero la presentación de esta nueva afección global nos obliga a expandir nuestro horizonte a la medicina crítica como lo es en Norteamérica (Estados Unidos y Canadá), ${ }^{5}$ la especialidad dual es uno de nuestros pendientes académicos. ${ }^{6}$

Esta rama médica tiene un impulso mediático y social sin precedentes en los últimos meses, es nuestra obligación que la misma perdure con nuestro trabajo y la preparación necesaria para el beneficio de los pacientes.

\section{Vita Longa Respirare}

\section{REFERENCIAS}

1. Rico Méndez FG. La neumología desde sus raíces. Un viaje a través del tiempo. México: Permanyer; 2018 [Accesado 24 de junio de 2020]. Disponible en: https://www.anmm.org.mx/publicaciones/ultimas_publicaciones/La-Neumologia-desde-raices.pdf 
2. Instituto Nacional de Enfermedades Respiratorias. [Accesado 25 de junio de 2020] Disponible en: https://www.gob.mx/salud/iner

3. Sandoval-Gutierrez JL, Reyes ES, Bautista EB. Pulmonary diseases: first cause of mortality in the world. Chest 2011;139(6):1550.

4. Organización Mundial de la Salud. Brote de enfermedad por coronavirus (COVID-19). 2020 [Accesado 26 de junio de 2020] Disponible en: https://www.who.int/es/emergencies/diseases/novel-coronavirus-
2019?gclid=EAlalQobChMltc34mfGa6gIVzMDACh1jKQRIEAAYASA AEglCufD_BwE

5. Richards JB, Spiegel MC, Wilcox SR. Characteristics of pulmonary critical care medicine and pulmonary medicine applicants and feIlowships. ATS Scholar 2020;1(1):67-77.

6. Sandoval Gutiérrez JL. Neumología-terapia intensiva como especialidad dual. Un binomio sin límites. Neumol Cir Torax 2010;69(3):178-179. 